\title{
Contrast Induced Nephropathy-A Collateral Damage to be Minimized
}

\author{
AAS Majumder, M Ullah \\ Department of Cardiology, NICVD, Dhaka.
}

(Cardiovasc. j. 2012; 5(1): 1-2)

With the increasing use of iodinated contrast medium $(\mathrm{CM})$ in diagnostic and interventional procedures, contrast-induced nephropathy (CIN) is the third most common cause of acute kidney injury. In the general population, the incidence of CIN is estimated to be $0.6 \%-2.3 \% .{ }^{1}$ However, the risk for developing CIN may be as high as $50 \%$ in some high-risk patients. CIN is associated with prolonged hospitalization, increased death rate, and increased cost. ${ }^{2}$ No current treatment can reverse or ameliorate CIN once it occurs, but prophylaxis is possible.Identification of high-risk patients and mitigation of the risk of CIN have become important and interrelated issues in cardiovascular medicine.

Hypotension (SBP $<80 \mathrm{mmHg}$ ), heart Failure (NYHA III/IV), use of intra-aortic balloon pump, preexisting renal dysfunction $(\mathrm{SCr}>1.5 \mathrm{mg} / \mathrm{dl} \mathrm{OR}$ $\mathrm{CrCl}<60 \mathrm{ml} / \mathrm{min}$ ), age $\geq 75$ years, diabetes, hematocrit $<39 \%$ for men, or $<36 \%$ for women, dehydration, concomitant use of nephrotoxic drugs and/or renal perfusion reducing agents (ACEI's, Aminoglycosides, Vancomycin, Diuretics, NSAID's, etc) are the risk factors for development of CIN. ${ }^{3}$ The Mehran CIN-Risk score was proposed as a simple risk score to be readily applied by clinicians to assess the individual risk for CIN development following percutaneous coronary intervention. ${ }^{4}$

To date, many prophylactic strategies have been investigated extensively. The recent guidelines have proposed the following strategies to prevent CIN-1) Hydration with intravenous infusion . 2) Use of low or iso-osmolar contrast medium and the lowest dose for at-risk patients. 3) Patients taking metformin should stop it. 4) Withdrawal of nephrotoxic drugs 5) Pharmacological prophylaxis.

Hydration is almost universally accepted as an appropriate and safe measure to prevent CIN. Two types of fluid can be used-a) normal saline b) sodium bicarbonate solution. The role sodium bicarbonate solution is controversial. Some of the studies has found it equal and in other studies it is inferior to normal saline infusion. ${ }^{3}$ Appropriate caution should be applied in the patient with known reduced left ventricular function or congestive heart failure. In a recent study it was found that oral hydration and alkalinization is noninferior to intravenous therapy for prevention of contrast-induced nephropathy in patients with chronic kidney disease. ${ }^{5}$

High osmolar contrast media increases the risk of CIN. But there was no significant difference between iso osmolar (iodixanol) and low osmolar (iopamidol) in either peak increase in $\mathrm{SCr}$ or risk of CIN ${ }^{6}$ Contrast media volume is an independent risk factor for CIN. Cigarroa et al ${ }^{7}$ reported that the maximum CM dose of lower osmolality to be administered to patients with reduced renal function is $1.5 \mathrm{ml} \times 5 \times \mathrm{kg}$ of body weight (maximum $300 \mathrm{ml}$ )) divided by serum creatinine (expressed in $\mathrm{mg} / \mathrm{dl}$ ). To lower the dose of $\mathrm{CM}$ a small size catheter, biplane or rotational coronary angiography may be used, and left ventriculography should be avoided in high-risk patients along with avoiding subsequent contrast media for 72 hours. Performing diagnostic and interventional procedures at separate sessions is appropriate if the clinical situation permits.

Contrast media induced renal vasoconstriction and oxidative stress are the important mechanisms of CIN, so a number of agents that improve renal vascular circulation, including endothelin antagonists, adenosine antagonists, atrial natriuretic peptide, dopamine agonists and calcium channel blockers, have been clinically investigated for the prevention of CIN. Most of them failed to 
prevent CIN in high-risk patients. Some antioxidants, including $\mathrm{N}$-acetylcysteine and ascorbic acid have also failed to show a consistent benefit in reducing the incidence of CIN when compared to volume repletion alone. ${ }^{8}$

Recently, the meta-analysis of prophylaxis using high dose statin for the prevention of CIN has been reported, indicating the support of the effectiveness of periprocedural short-term treatment with highdose statin. ${ }^{9}$ But it needs further large randomized clinical trial.

Immediately after angiography, hemodialysis is often performed to remove the contrast medium in patients with advanced renal dysfunction. But in the study of Morcos et al, hemodialysis did not protect against CIN. ${ }^{10}$ Therefore, many of the guidelines do not recommend it. Because by the time hemodialysis is done, contrast media has already passed to the kidneys and has done the harm.

Patients treated with primary PCI may represent a population at higher risk for CIN than those undergoing elective PCI. Several conditions may contribute to renal injury in this setting. Among them, hypotension or even shock, a large volume of contrast media, and the impossibility of starting a renal prophylactic therapy are the factors most likely involved.

Rapid treatment of cardiogenic shock, minimization of contrast volume administered, treatment of dehydration, and, ostensibly, early and rapid hydration is all-important. Early and rapid hydration should be continued for 12 hours to a mean volume of almost $1200 \mathrm{~mL} .^{11}$

CIN is a common complication, particularly in highrisk patients. Pericatheterization hydration, discontinuation of potentially nephrotoxic drugs, and using the lowest possible dose of contrast media are the methods by which we can effectively reduce the incidence of CIN. However, further studies are essential to find out new agents in preventing CIN.

\section{References:}

1. Vercellino M, Bezante GP, Balbi M. Contrast medium induced nephropathy: new insights into prevention and risk management. Cardiovasc Hematol Agents Med Chem 2009; 7: 166-180.

2. Solomon RJ, Mehran R, Natarajan MK, Doucet S, Katholi RE, Staniloae CS, et al. Contrast-induced nephropathy and long-term adverse events: cause and effect? Clin J Am Soc Nephrol 2009; 4: 1162-1169.

3. Pannu N, Wiebe N, Tonelli M. Prophylaxis strategies for contrast-induced nephropathy. JAMA 2006; 295: 276579 .

4. Mehran R, Aymong ED, Nikolsky E, et al. A simple risk score for prediction of contrast-induced nephropathy after percutaneous coronary intervention: development and initial validation. J Am Coll Cardiol 2004; 44: 1393-1399.

5. Cho R, Javed N, Traub D, Kodali S, Atem F, Srinivasan V. Oral hydration and alkalinization is noninferior to intravenous therapy for prevention of contrast-induced nephropathy in patients with chronic kidney disease. $J$ Interv Cardiol 2010; 5 : 460-466.

6. Richard J. Solomon, Madhu K. Natarajan, Serge Doucet, Samin K. Sharma, Cezar S. Staniloae, Richard E. Katholi, Joseph L. Gelormini, Marino Labinaz, Abel E. Moreyra, the Investigators of the CARE Study. Cardiac Angiography in Renally Impaired Patients (CARE) Study. Circulation 2007;115:3189-3196.

7. Cigarroa RG, Lange RA, Williams RH, Hillis LD. Dosing of contrast material to prevent contrast nephropathy in patients with renal disease. Am J Med 1989; 86: 649-652.

8. Holger Thiele, Lysann Hildebrand, Carmen Schirdewahn, Ingo Eitel, Volker Adams, Georg Fuernau, Sandra Erbs, Axel Linke, Klaus-Werner Diederich, Marek Nowak, Steffen Desch, Matthias Gutberlet, Gerhard Schuler. Impact of High-Dose NAcetylcysteine Versus Placebo on Contrast-Induced Nephropathy and Myocardial Reperfusion Injury in Unselected Patients With ST-Segment Elevation Myocardial Infarction Undergoing Primary Percutaneous Coronary Intervention - The LIPSIA-NACC trial. J Am Coll Cardiol 2010;55:2201-2209.

9. Zhang BC, Li WM, Xu YW. High-dose statin pretreatment for the prevention of contrast-induced nephropathy: A meta-analysis. Can J Cardiol 27: 851858, 2011.

10. Rodby RA. Preventing complications of radiographic contrast media: is there a role for dialysis? Semin Dial 2007; 20: 19-23.

11. Charanjit S. Rihal, Kianoush B. Kashani. Intravascular Volume Expansion Before Primary Angioplasty for Prevention of Acute Kidney Injury Hydration or Dilution? Circ Cardiovasc Interv 2011;4:405-406. 